\title{
Clinical, Diagnostic and Therapeutic Approaches to Complications of Brucellosis: An Experience of 12 Years
}

\author{
Gülşen Mermut Onur Özgenç Meltem Avcı Ali llgın Olut Ebru Öktem \\ Vecdi Evren Genç Alpay Arı Seher Ayten Coskuner \\ Department of Infectious Diseases and Clinical Microbiology, Izmir Bozyaka Training and Research Hospital, \\ Izmir, Turkey
}

\section{Key Words}

Brucellosis · Complications • Clinical symptoms ·

Diagnosis • Treatment

\section{Abstract}

Objective: To describe the clinical presentations, laboratory findings, prevalence and pattern of complications and the response to treatment of brucellosis in a 12-year period in a Turkish research hospital. Materials and Methods: Between 1996 and 2008, 231 patients were diagnosed with brucellosis and treated in our clinic. Medical records of 189 of the 231 patients with at least one demonstrable complication of the disease were reviewed for anamnesis, diagnosis, complications, treatment and clinical outcomes. Results: The decreasing order of the complications was: hematological, 104 (55\%); osteoarticular, 70 (37\%); hepatobiliary, 59 (31\%), and gastrointestinal, 23 (12\%). The most common laboratory findings were anemia, lymphomonocytosis, elevated sedimentation rate and C-reactive protein, and elevated aminotransaminases. Conclusion: The hematological, osteoarticular and hepatobiliary manifestations were predominant. Bur-
\end{abstract}

sitis, synovitis, glomerulonephritis, cutaneous lesion and deep vein thrombosis were the rare complications observed in our study. In clinical practice, brucellosis should be considered in the differential diagnosis in the presence of infrequent complications.

Copyright @ 2011 S. Karger AG, Basel

\section{Introduction}

Brucellosis is a systemic disease that may involve any organ or system in the body and may mimic various multisystem diseases showing a wide clinical polymorphism. Clinically it may progress as acute, subacute or chronic infection, and as Brucella spp. are intracellular bacteria that can survive and multiply in phagocytic cells of the hosts, relapses may be seen. Complications of the disease are relatively common and widespread; the most common affected systems are the osteoarticular, gastrointestinal, hematological, genitourinary, cardiovascular, respiratory and central nervous systems [1-4].

\section{KARGER}

Fax +4161306 1234

E-Mail karger@karger.ch

www.karger.com
(C) 2011 S. Karger AG, Basel

1011-7571/12/0211-0046\$38.00/0

Accessible online at:

www.karger.com/mpp
Meltem Avc1

Department of Infectious Diseases and Clinical Microbiology

Izmir Bozyaka Training and Research Hospital

Izmir (Turkey)

Tel. +90 532634 9818, E-Mail meltema1@ hotmail.com 
In this report, we describe the clinical presentations, laboratory findings, prevalence and pattern of complications and the response to treatment of brucellosis in a 12-year period in a Turkish research hospital.

\section{Materials and Methods}

Between 1996 and 2008, 231 patients were diagnosed with brucellosis and treated in our clinic of infectious diseases. In 189 patients, at least one complication of the disease was demonstrated; their medical records were reviewed for anamnesis, diagnosis, complications, treatment and clinical outcomes. The diagnosis was made by isolation of Brucella spp. either in blood, bone marrow, cerebrospinal fluid, operation material and/or compatible clinical features such as fever, arthralgia, sweating, chills, malaise supported by a positive rose bengal test plus a positive standard agglutination test. All patients had a complete blood count, sedimentation rate, $\mathrm{C}$-reactive protein, blood chemistry profiles and urine analysis. All patients had blood cultures (automated blood culture system Bactec 9240, Becton-Dickinson Diagnostic Instrument System, Franklin Lakes, N.J., USA). Other cultures done were those of cerebrospinal fluid, bone marrow or biopsies taken during surgery depending on the patient's clinical findings.

Complications of the disease were determined and grouped according to the organ or system involved as: osteoarticular, hematological, hepatobiliary, gastrointestinal, urogenital, cardiovascular, neurological, respiratory and cutaneous [1].

Osteoarticular involvement was considered if there were any signs of inflammation (e.g. pain, redness, heat, swelling, restriction of movement) in any joint and/or radiographic abnormalities. Computed tomography or magnetic resonance imaging was used especially to detect sacroiliitis, vertebral osteomyelitis, spondylodiscitis, paraspinal abscess, bursitis and synovitis. Hematological complications were defined as laboratory abnormalities such as anemia, leukopenia, thrombocytopenia or presence of any clinical signs of a coagulation disorder. Hepatobiliary involvement was defined as a more than 2-fold increase in aminotransferase levels without any other etiological explanation and/or serum total bilirubin levels $>2.5 \mathrm{~g} / \mathrm{dl}$. Gastrointestinal complications were considered if there were any signs or symptoms of involvement such as nausea/vomiting, diarrhea, constipation or abdominal tenderness. Neurological manifestations of brucellosis were either from direct invasion of the bacteria such as meningitis and abscess or changes in mental or mood status. In suspected patients, cardiovascular complications were detected by either transesophageal echocardiography or Doppler ultrasonographic analysis.

Most of the patients had been treated with either one of the three following antibiotic regimens: doxycycline $(200 \mathrm{mg} /$ day $)+$ streptomycin (1 g/day), doxycycline (200 mg/day) + rifampin (600 $\mathrm{mg}$ /day) or doxycycline $(200 \mathrm{mg} /$ day $)+$ rifampin $(600 \mathrm{mg} /$ day $)+$ streptomycin (1 g/day). The duration of therapy was at least 6 weeks, but in some cases it was longer, due to the nature of complications or delayed clinical, laboratory or radiological responses. The mean duration of therapy in all patients was $75 \pm 57$ days (45-365 days). Patients were recalled to follow-up visits in the 2 nd, 4 th, 12 th and 24 th weeks after treatment for clinical examination and laboratory tests. Relapse was defined as reappearance of clinical symptoms either with or without blood culture positivity.

Complications of Brucellosis

\section{Results}

The mean age of the 189 patients with complications of brucellosis was $44.8 \pm 16.3$ years (range 15-77 years), and $105(56 \%)$ of them were men. In $68(36 \%)$ patients no risk factors or possible routes of transmission could be found. The most common risk factor was the consumption of unpasteurized milk or dairy products in $88 \mathrm{pa}-$ tients (47\%), and in $33(17 \%)$ there was an occupational risk factor such as being a farmer, butcher or veterinarian. The most common presenting symptom of the disease was fever $>38.3^{\circ} \mathrm{C}$ in 125 cases (66\%) followed by arthralgia in $72(38 \%)$, back pain in $68(36 \%)$ and malaise in 66 (35\%). Anemia was present in $64(34 \%)$ patients with a mean hemoglobin level of $10.8 \pm 1.6 \mathrm{~g} / \mathrm{dl}$. The sedimentation rate was elevated in $138(73 \%)$ patients with a mean rate of $39 \pm 16 \mathrm{~mm} / \mathrm{h}$. Hepatomegaly was present in 33 (18\%) patients on presentation, and serum aminotransferases were elevated in 59 (31\%) patients with a mean of $62 \pm 17 \mathrm{IU} / \mathrm{l}$ (normal range $\leq 32$ ). All of the patients were positive for rose bengal or standard agglutination tests and a mean titer of $1 / 480$ (range 1/80-1/1,280). Clinical signs/symptoms of the disease and the laboratory findings are presented in tables 1 and 2 .

Hematological complications in 104 patients (55\%) were the leading types of the complications followed by osteoarticular $(70 ; 37 \%)$, hepatobiliary $(59 ; 31 \%)$ and gastrointestinal ones $(23 ; 12 \%)$. The most common joints involved were the vertebral, followed by the peripheral and sacroiliac joints. Spondylodiscitis was present in 48 (26\%), peripheral arthritis in $10(5 \%)$ and sacroiliitis in $8(4 \%)$ patients with complications. In 16 patients with spondylodiscitis, abscess formation was seen in the paravertebral or epidural area, and 6 patients had also concomitant sacroiliac joint involvement. Genitourinary complications were present in 9 (5\%), in which 8 patients had unilateral epididymo-orchitis and 1 had glomerulonephritis. Cardiovascular involvement was present in 2 (1\%) patients. One of them had endocarditis of the aortic valve, and the other had deep venous thrombosis, a rarely reported complication of brucellosis. The central nervous system was affected in $9(5 \%)$ patients: meningitis 6, brain abscess 1 , and major depressions 2 . One patient had a petechial rash that resolved within 2 weeks after treatment had been started. The distributions of complications are summarized in table 3.

In our clinic, 182 (96\%) of the brucellosis cases were treated with either one of the three following regimens: doxycycline + streptomycin, 100 (52.5\%); doxycycline + rifampin + streptomycin, $56(30 \%)$, and doxycycline + ri- 
Table 1. Epidemiology, symptoms and signs of patients with complicated Brucella infection

\begin{tabular}{|c|c|c|}
\hline Variable & Number & Percent \\
\hline \multicolumn{3}{|l|}{ Sex } \\
\hline Female & 84 & 44 \\
\hline Male & 105 & 56 \\
\hline \multicolumn{3}{|l|}{ Possible route of transmission } \\
\hline Unpasteurized dairy products & 88 & 47 \\
\hline Occupational & 33 & 17 \\
\hline Unknown & 68 & 36 \\
\hline \multicolumn{3}{|l|}{ Clinical type } \\
\hline Acute & 109 & 58 \\
\hline Subacute & 64 & 34 \\
\hline Chronic & 15 & 8 \\
\hline \multicolumn{3}{|l|}{ Symptoms } \\
\hline Fever $\left(>38.3^{\circ} \mathrm{C}\right)$ & 125 & 66 \\
\hline Arthralgia & 72 & 38 \\
\hline Lumbar pain & 68 & 36 \\
\hline Malaise & 66 & 35 \\
\hline Sweating & 28 & 15 \\
\hline Myalgia & 23 & 12 \\
\hline Diarrhea or constipation & 23 & 12 \\
\hline Weight loss & 18 & 10 \\
\hline Headache & 10 & 5 \\
\hline Lack of appetite & 2 & 1 \\
\hline \multicolumn{3}{|l|}{ Signs } \\
\hline Hepatomegaly & 33 & 18 \\
\hline Splenomegaly & 32 & 17 \\
\hline Hepatosplenomegaly & 15 & 8 \\
\hline Lymphadenopathy & 7 & 4 \\
\hline
\end{tabular}

fampin, 26 (14\%). In 7 patients either ceftriaxone, trimethoprim-sulfamethoxazole or ciprofloxacin was used in combination with doxycycline or rifampin. Therapeutic regimens and relapse rates of cases are summarized in table 4 .

\section{Discussion}

The predominant complications of brucellosis in this study were hematological, osteoarticular and hepatobiliary manifestations similar to previous reports $[1,3,5-8]$; the osteoarticular complications of $37 \%$ of this study are within the range of $10-80 \%$ reported previously [1]. The most common joint involved was the vertebral joint, in $69 \%$ of all patients with osteoarticular involvement, followed by peripheral and sacroiliac joints. More than one third of the spondylodiscitis cases (33.3\%) were complicated by an abscess formation in the paravertebral or epi-
Table 2. Laboratory findings of patients with complicated Brucella infection

\begin{tabular}{lrc}
\hline Laboratory findings & Number & Percent \\
\hline Anemia & 64 & 34 \\
Leucopenia & 16 & 8 \\
Leukocytosis & 4 & 2 \\
Thrombocytopenia & 8 & 4 \\
Anemia + leukopenia & 2 & 1 \\
Pancytopenia & 10 & 5 \\
Lymphomonocytosis & 81 & 43 \\
Elevated C-reactive protein & 128 & 68 \\
Elevated sedimentation rate & 138 & 73 \\
$\quad 20-40$ mm/h & 82 & 43 \\
$\quad$ >40 mm/h & 56 & 30 \\
Elevated aminotransferases & 59 & 31 \\
Blood culture positivity & 61 & 32 \\
Rose bengal positivity & 189 & 100 \\
Standard agglutination test positivity & 189 & 100 \\
\hline
\end{tabular}

Table 3. Complications of brucellosis

\begin{tabular}{lrl}
\hline & Number & Percent \\
\hline Hematological & 104 & 55 \\
Osteoarticular & 70 & 37 \\
$\quad$ Spondylodiscitis & 48 & 26 \\
Sacroiliitis & 8 & 4 \\
Peripheral arthritis & 10 & 5 \\
Bursitis & 2 & 1 \\
Synovitis & 2 & 1 \\
Hepatobiliary & 59 & 31 \\
Gastrointestinal & 23 & 12 \\
Genitourinary & 9 & 5 \\
$\quad$ Epididymo-orchitis & 8 & 4 \\
Glomerulonephritis & 1 & 0.5 \\
Central nervous system & 9 & 5 \\
$\quad$ Meningitis & 6 & 3.5 \\
$\quad$ Brain abscess & 1 & 0.5 \\
Major depression & 2 & 1 \\
Cardiovascular system & 2 & 1 \\
Endocarditis & 1 & 0.5 \\
Deep vein thrombosis & 1 & 0.5 \\
Skin & 1 & 0.5 \\
\hline
\end{tabular}

dural area. Patients with osteoarticular complications were treated with doxycycline + streptomycin with or without addition of rifampin, for 3-12 months. The response to the doxycycline + rifampin + streptomycin combination seemed to be better, as the relapse rate was 
Table 4. Initial therapeutic regimens according to patients and relapse rates

\begin{tabular}{lrrr}
\hline Therapeutic regimen & Patients & \multicolumn{2}{c}{ Relapses } \\
\cline { 3 - 4 } & & $\mathrm{n}$ & $\%$ \\
\hline Positive osteoarticular involvement & 70 & 6 & 8 \\
Doxycycline + streptomycin & 28 & 3 & 11 \\
Doxycycline + rifampin + streptomycin & 42 & 3 & 7 \\
Doxycycline + rifampin & 0 & & \\
\hline Other patients & 119 & 6 & 5 \\
Doxycycline + streptomycin & 72 & 3 & 4 \\
Doxycycline + rifampin + streptomycin & 14 & 1 & 7 \\
Doxycycline + rifampin & 26 & 2 & 7 \\
Other & 7 & 0 & \\
\hline
\end{tabular}

$7 \%$ compared with $11 \%$ in patients treated with doxycycline + streptomycin.

The 55\% hematological complications of our study are similar to the $56 \%$ reported by Dilek et al. [5]. Anemia was the most common hematological complication (34\%), with a mean hemoglobin level of $10.8 \pm 1.6 \mathrm{~g} / \mathrm{dl}$. Pancytopenia was present in $5 \%$ of patients but no serious complications such as a clotting disorder or disseminated intravascular coagulation were seen.

Cardiovascular complications are rare but most serious complications of brucellosis as only 2 patients of our study showed evidence of cardiovascular involvement. One had aortic valve endocarditis, which was treated with the doxycycline + rifampin + ciprofloxacin combination for 6 months, and the other presented with deep venous thrombosis. Rare but serious cardiovascular complications have been reported by others $[1,3,6,7,9,10]$. Some of these cases involved endocarditis and aortic valves; however, myocarditis and mycotic aneurysms of vessels are secondary complications $[1,3,6]$.

The $3.7 \%$ of direct invasion of the central nervous system of our study is slightly less than the $5-6.6 \%$ reported in other studies $[1,3,11,12]$. Acute or chronic meningitis is the most common manifestation of neurobrucellosis but meningoencephalitis, brain abscess, epidural abscess and demyelinating disorders and meningovascular syndromes have also been reported. Of 7 patients (3.7\%) of our study who manifested a direct invasion of the central nervous system, 6 had meningitis and the other had a brain abscess. The patients with meningitis were treated with a triple drug combination, with doxycycline + rifampin + either ceftriaxone or trimethoprim-sulfa- methoxazole, for 3-6 months. The brain abscess required surgical removal despite antibiotic treatment.

Involvement of the genitourinary system occurred in $5 \%$ of patients in this study. Of 9 patients, 8 had epididymo-orchitis and the other had glomerulonephritis, which is a rarer complication. It is reported that epididymo-orchitis can be seen in up to $20 \%$ of men [1]. As epididymoorchitis is a rare cause of testicular mass, brucellosis should be kept in mind in the differential diagnosis of this situation to avoid unnecessary orchiectomy [13].

Although cutaneous findings such as rashes, ulcers, abscess, erythema nodosum, petechiae, purpura, vasculitis and contact dermatitis have been reported in about $5 \%$ of patients [1], in this study only 1 patient had a cutaneous lesion that was a petechial rash which disappeared within 2 weeks after treatment had been started.

As defined in many reports $[1,3]$, we also found an elevated sedimentation rate and C-reactive protein, lymphomonocytosis, anemia and elevated aminotransaminases as the most common laboratory findings. The $32 \%$ of positive blood cultures found in this study are within the $15-90 \%$ range reported in previous studies $[1,14]$. Differences in isolation rates between centers may be due to delay in diagnosis and previous antibiotic usage of the patients before admission to clinics.

\section{Conclusion}

The hematological, osteoarticular and hepatobiliary manifestations were predominant complications while bursitis, synovitis, glomerulonephritis, cutaneous lesion and deep vein thrombosis were rare complications observed in our study. In clinical experience, brucellosis should be considered in the differential diagnosis in the presence of infrequent complications.

References

1 Young EJ: Brucella species; in Mandell GL, Bennet JE, Dolin R (eds): Principles and Practice of Infectious Diseases, ed 7. Philadelphia, Churchill Livingstone, 2010, pp 2921-2925.

-2 Franco MP, Mulder M, Gilman RH, Smits HL: Human brucellosis. Lancet Infect Dis 2007;7:775-786

- 3 Buzgan T, Karahocagil MK, Irmak H, Baran AI, Karsen H, Evirgen O, Akdeniz H: Clinical manifestations and complications in 1,028 cases of brucellosis: a retrospective evaluation and review of the literature. Int J Infect Dis 2010;14:469-478. 
4 Al-Anazi AR, Aziz S, Fouda MA: Brucellosis: haemorrhagic pleural effusion. Med Princ Pract 2005;14:118-120.

5 Dilek I, Durmus A, Karahocagil MK, Akdeniz H, Karsen H, Baran AI, Evirgen O: Hematological complications in 787 cases of acute brucellosis in Eastern Turkey. Turk J Med Sci 2008;38:421-424.

-6 Aygen B, Doğanay M, Sümerkan B, Yildız O, Kayabas Ü: Clinical manifestations, complications and treatment of brucellosis: a retrospective evaluation of 480 patients. Med Mal Infect 2002;32:485-493.
7 Akdeniz H, Irmak H, Demiröz AP: Evaluation of brucellosis cases in Van region of Eastern Anatolia: a-3 year experience. Nagoya Med J 1998;42:101-110.

8 Pamuk GE, Celik AD, Uyanık MS: Brucellosis triggering hemolytic anemia in glucose-6-phosphate dehydrogenase deficiency. Med Princ Pract 2009;18:329-331.

9 Ertek M, Yazgi H, Kadanali A, Özden K, Taşyaran MA: Complications of Brucella infection among adults: an 18 year retrospective evaluation. Turk J Med Sci 2006;36:377381.

10 Odeh M, Pick N, Oliven A: Deep venous thrombosis associated with acute brucellosis. Angiology 2000;51:253-256.
11 Haji-Abdolgabi M, Rasooli-Nejad M, Jafari S, Hasibi M, Soudbakhsh A: Clinical and laboratory findings in neurobrucellosis: review of 31 cases. Arch Iranian Med 2008;11:21-25.

12 Yetkin MA, Bulut C, Erdinc FS, Oral B, Tulek $\mathrm{N}$ : Evaluation of the clinical presentations in neurobrucellosis. Int J Infect Dis 2006;10: 446-452.

13 Hizli F, Uygur MC: Brucella orchitis: a rare cause of testicular mass - report of a case. Int Urol Nephrol 2006;38:637-639.

14 Dimitrov TS, Panigrahi D, Emara M, Awni F, Passadilla R: Seroepidemiological and microbiological study of brucellosis in Kuwait. Med Princ Pract 2004;13:215-219. 